\title{
Relation entre Maladies Parodontales et Maladies cardiovasculaires : Revue de la littérature.
}

\author{
Relationship between Periodontal Diseases and Cardiovascular Diseases: A review of \\ Literature.
}

TOGO AK ${ }^{1}$, KANE AST $^{2}$, TRAORE $\mathrm{H}^{3}$, DIABY LM ${ }^{2}$, SANOGO A ${ }^{2}$, DIAWARA O ${ }^{3}$, DIOP A ${ }^{4}$.

${ }^{1}$ Polyclinique Mohamed 5 (MALI) ; ${ }^{2}$ Service d'Odontologie, Infirmerie Hôpital Militaire de Bamako IHB

(MALI) $;{ }^{3} \mathrm{CHU}$ Odontostomatologie de Bamako (Mali) ; ${ }^{4}$ Service de Chirurgie Maxillo-faciale et stomatologie, Hôpital National Donka, CHU Conakry (GUINEE).

Correspondance : Dr Abdoul Karim TOGO, Chirurgien-dentiste à la Polyclinique Mohamed 5 (Mali).

Email : aktogo@yahoo.fr, Bamako / MALI.

\section{RESUME}

Introduction : La maladie parodontale (MP) est une maladie inflammatoire complexe qui détruit les tissus de soutien de la dent (parodonte) avec une étiologie multifactorielle elle a été liée au diabète, aux obstructions chroniques des voies respiratoires, aux maladies rénales chroniques, aux syndromes métaboliques, à certains types de cancers, la polyarthrite rhumatoïde et aux maladies cardiovasculaires. Les maladies cardiovasculaires (MCV) constituent un ensemble de troubles affectant le cœur et les vaisseaux sanguins, leur cause la plus courante est l'athérosclérose, qui correspond à un remaniement pathologique de la paroi interne des artères de gros et moyen calibres par accumulation de lipides, glucides complexes, éléments sanguins et dépôts calcaires, formant des plaques d'athérosclérose. Ainsi, l'objectif de cette revue de la littérature est de déterminer la relation entre les maladies parodontales et les maladies cardiovasculaires. Matériels et Méthodes : Pour ce travail une stratégie de recherche documentaire électronique a été réalisée dans 2 bases de données dont Pub Med et Google scholar. Ainsi 423 articles traitant la relation entre les maladies parodontales et les maladies cardiovasculaires publiés en anglais ou en français avec texte intégral dans la période allant de 1989 à 2016 ont été sélectionnés. Les titres et résumés des articles retrouvés grâce à la stratégie de recherche ont été passés en revue. Les articles qui n'étaient pas pertinents pour une inclusion ont été écartés dès ce stade. Les données recueillies ont subi une analyse après mise en œuvre des critères d'inclusions : Etudes réalisées chez les humains et rédigées en anglais ou en français et publiés entre 1989 à 2016. Les articles inclus étaient ceux après lecture des titres, des résumés jugés pertinents dont 35 articles traitant la relation entre les maladies parodontales et les Maladies Cardiovasculaires ont été sélectionnés. Résultat : Au total 35 articles traitant la relation entre les maladies parodontales et les Maladies cardiovasculaires ont été sélectionnés compte tenu de leurs cohérence et pertinence. La limite a été la consultation que de 2 bases de données. Les bactériémies associées aux parodontites sont responsables d'une activation de la réponse immuno-inflammatoire favorisant la formation, la maturation et la complication des plaques d'athérome. Il existe un risque accru de MCV chez les patients atteints de parodontite chronique, indépendamment des autres facteurs de risques cardiovasculaires. Le traitement parodontal réduit le niveau d'inflammation systémique et améliore la fonction endothéliale, mais ne modifie pas la dyslipidémie. Conclusion: avec une étiologie infectieuse pour les maladies et divers pour les maladies cardiovasculaires, un lien a été établi entre les deux maladies dans diverses études épidémiologiques et cliniques mais toutes fois reste controverse dans d'autre. Mots clés : Relation, Parodontites, Maladie cardiovasculaires, Revue.

\section{ABSTRACT}

Introduction: Periodontal disease (PD) is a complex inflammatory disease that destroys tooth support tissue (periodontal) with a multifactorial etiology it has been linked to diabetes, chronic obstructions of the respiratory tract, chronic kidney disease, syndromes certain types of cancers, rheumatoid arthritis and cardiovascular diseases. Cardiovascular disease (CVD) is a group of disorders affecting the heart and blood vessels, the most common cause is atherosclerosis, which is a pathological reworking of the inner lining of large and medium-sized arteries by lipid accumulation complex carbohydrates, blood elements and calcareous deposits, forming plaques of atherosclerosis. Thus, the purpose of this review of the literature is to determine the relationship between periodontal disease and cardiovascular disease. Materials and Methods: For this work an electronic document search strategy was realized in 2 databases including Pub Med and Google scholar. Thus 423 articles dealing with the relation between periodontal diseases and cardiovascular diseases published in English or in French with full text in the period from 1989 to 2016 were selected. The titles and abstracts of the articles found thanks to the search strategy were reviewed. Articles that were not relevant for inclusion were discarded at this stage. The data collected were analyzed after implementation of the inclusion criteria: Studies conducted in humans and written in English or French and published between 1989 and 2016. 
Included articles were those after reading titles, summaries deemed relevant of which 35 articles dealing with the relationship between periodontal diseases and Cardiovascular Diseases were selected. Result: A total of 35 articles dealing with the relationship between periodontal disease and cardiovascular disease were selected based on their consistency and relevance. The limit has been the consultation only of 2 databases. The bacteremia associated with periodontitis is responsible for an activation of the immuno-inflammatory response favoring the formation, maturation and complication of atheroma plaques. There is an

\section{INTRODUCTION}

La maladie parodontale (MP) est une maladie inflammatoire complexe qui détruit les tissus de soutien de la dent (parodonte) avec une étiologie multifactorielle elle a été liée au diabète, aux obstructions chroniques des voies respiratoires, aux maladies rénales chroniques, aux syndromes métaboliques, à certains types de cancers, la polyarthrite rhumatoïde et aux maladies cardiovasculaires. Les MP sont très répandues et peuvent affecter jusqu'à $90 \%$ de la population avec un degré variable de graviter de la maladie [1]. Les maladies cardiovasculaires (MCV) constituent un ensemble de troubles affectant le cœur et les vaisseaux sanguins, leur cause la plus courante est l'athérosclérose, qui correspond à un remaniement pathologique de la paroi interne des artères de gros et moyen calibres par accumulation de lipides, glucides complexes, éléments sanguins et dépôts calcaires, formant des plaques d'athérosclérose. Selon l'Organisation Mondiale de la Santé, les MCV sont la première cause de mortalité dans le monde, elles représentent 17,3 millions de décès, soit $30 \%$ de la mortalité mondiale totale [1]. Près de $42 \%$ des décès par MCV sont liés aux cardiopathies coronariennes et $34 \%$ aux maladies cérébrovasculaires. Plus de $80 \%$ des décès interviennent dans des pays à revenu moyen ou faible et touchent presque également les hommes et les femmes [1]. D'ici 2030, près de 23,6 millions de personnes mourront d'une MCV, la grande majorité étant une complication de l'athérosclérose. D'après les projections, ces maladies devraient rester les premières causes de décès au niveau mondial [1]. Les MCV et les MP présentent différents mécanismes étiologiques, diverses pour les MCV et infectieuses pour les MP. Un lien entre les deux maladies a été établi dans de nombreuses études cliniques et épidémiologiques. Depuis, des métaanalyses ont confirmé que le risque de survenue de MCV est significativement augmenté de 14\% (pour les études prospectives) à $122 \%$ (pour les études cas-contrôles) chez les sujets atteints d'une maladie parodontale $[2,3,4,5]$. increased risk of CVD in patients with chronic periodontitis, independent of other cardiovascular risk factors. Periodontal therapy reduces the level of systemic inflammation and improves endothelial function, but does not affect dyslipidemia. Conclusion: With an infectious etiology for diseases and various for cardiovascular diseases, a link has been established between the two diseases in various epidemiological and clinical studies but all the time remains controversial in others. Key words: Relation, Periodontitis, Cardiovascular disease, review.

Ainsi, l'objectif de cette revue de la littérature est de déterminer la relation entre les maladies parodontales et les maladies cardiovasculaires.

\section{MATERIELS ET METHODES}

Pour ce travail une stratégie de recherche documentaire électronique a été réalisée dans 2 bases de données dont Pub Med et Google scholar. Ainsi 423 articles traitant la relation entre les maladies parodontales et les maladies cardiovasculaires publiés en anglais ou en français avec texte intégral dans la période allant de 1989 à 2016 ont été sélectionnés. Ont été sélectionnés les articles traitant la relation entre les maladies parodontales et les maladies cardiovasculaires en utilisant les mots clés suivants, Maladies parodontales, Prévalence des parodontites, Mécanismes des MCV, Facteurs de risque publiés en anglais ou français avec résumé ou texte intégral dans la période allant de 1989 à 2016. Comme critères de choix des articles, n'ont pas été inclus ceux traités moins pertinents ainsi que les lettres à la rédaction et les commentaires. Les articles inclus étaient ceux après lecture des titres, des résumés des articles et leurs analyses. L'échantillon de 35 articles traitant la relation entre les maladies parodontales et les Maladies Cardiovasculaires ont été sélectionnés selon nos critères d'inclusion.

\section{RESULTAT}

$\mathrm{Au}$ total 35 articles traitant la relation entre les maladies parodontales et les Maladies cardiovasculaires ont été sélectionnés compte tenu de leurs cohérence et pertinence. Sur les 35 articles inclus, 23 articles proviennent de PubMed/Medline et 12 de Google Scholar et 29 étaient en anglais. La limite a été la consultation que de 2 bases de données ainsi que la recherche manuelle (livres, thèses, mémoires) n'a pas été effectuée. Les relations obtenues pertinentes des différentes études sont basées sur deux mécanismes majeurs, immuno-inflammatoire (indirect) et bactérien (direct). Les maladies bucco-dentaires chroniques peuvent augmenter le risque de maladie coronarienne et peuvent constituer un facteur de risque non conventionnel pour la maladie 
coronarienne [32]. Certains espèces telles que Porphyromonas gingivalis (Pg), Treponema denticola (Td), mutans et A. Actinomycetecomitans, sont liées à la bactériémie et peuvent être des facteurs étiologiques pour le développement des maladies cardiovasculaires [29-34].

\section{DISCUSSION}

En 1989, Mattila et al. [6] ont publié une étude devenue principe, établissant un lien entre santé dentaire et survenue d'Infarctus du Myocarde IDM. Depuis, des méta-analyses ont confirmé que le risque de survenue de MCV est significativement augmenté de $14 \%$ (pour les études prospectives) à $122 \%$ (pour les études cas-contrôles) chez les sujets atteints d'une maladie parodontale [2,3,4,5]. Toutefois, selon l'American heart Association, si l'association entre les maladies parodontales et les MCV est indépendante des autres facteurs de risque, il n'est pas encore établi de lien causal après 30 ans de recherche et la puissance du lien apparait faible [7]. Enfin, l'association positive entre maladies parodontales et MCV varie selon le sexe et les tranches d'âges étudiées, et ne semble pas être retrouvée pour les sujets âgés de plus de 65 ans [8]. Les maladies parodontales sont à l'origine de bactériémies et d'endotoxémies transitoires. Dès lors, les effets à distance de ces expositions répétées sont biologiquement possibles. Deux mécanismes majeurs, immuno-inflammatoire (indirect) et bactérien (direct), sont actuellement avancés et peuvent coexister. Les maladies parodontales pourraient provoquer une réaction immunoinflammatoire se traduisant par la production de médiateurs et marqueurs pro-inflammatoires : CRP, interleukines, fibrinogène, matricielles (MMP), etc.. , par la production d'espèces réactives de l'oxygène (stress oxydant) et de facteurs prothrombotique par l'aggravation de la dyslipidémie, par des réactions anticorps-antigènes croisées, notamment les heart shock proteins (HSP) bactériennes et humaines, et enfin par une susceptibilité génétique partagée avec les MCV, favorisant le développement de l'athérosclérose de façon indirecte [9]. Les bactéries parodontales, en se greffant directement à une lésion vasculaire localisée ou à l'endothélium active, pourraient favoriser le recrutement des leucocytes (neutrophiles) et participer directement à l'athérogenèse en impactant l'évolution vers des complications cliniques comme la rupture de la plaque d'athérome [10,11]. S'il est clair que la réaction immuno-inflammatoire induite par les maladies parodontales augmente le niveau systémique des médiateurs inflammatoires impliqués dans l'athérogène, il manque des preuves pour dire que cette augmentation influence réellement le développement et/ou la progression de l'athérosclérose et de ses conséquences cliniques (IDM, maladies cérébrovasculaires) [9]. La deuxième hypothèse se fonde sur les preuves accumulées chez l'homme et chez l'animal (études in vivo et in vitro) que l'invasion des tissus vasculaires par les bactéries parodontales participe au développement de la maladie athéromateuse. Les parodontopathogènes majeurs ont la capacité de gagner les sites vasculaires via différents mécanismes : la transcytose, les brèches gingivales iatrogènes (détartrage- surfaçage, avulsion, sondage) ou quotidiennes (mastication, brossage, fil dentaire) et, enfin, via l'internalisation dans les leucocytes. Ces bactériémies sont corrélées à l'état parodontal (indice de plaque et indice gingival) [12]. La présence d'ADN de nombreuses espèces de bactéries parodontales au sein de différents tissus vasculaires [12,14] a été montrée dans de nombreuses études. Certaines études suggèrent que Porphyromonas gingivalis et Aggregatibacter Actinomycetecomitans pourraient survivre dans les plaques d'athérome [15]. Une étude rapporte même la culture de $P$. gingivalis à partir d'endartériectomies carotidiennes, en présence de monocytes [16]. D'autres études utilisent les anticorps dirigés contre les parodontopathogènes majeurs pour détecter de façon indirecte leur présence via la réponse humorale induite chez les patients. L'augmentation des taux sériques d'anticorps anti- $P$. gingivalis et anti-A. Actinomycetecomitans est associée au risque de maladies cérébrovasculaires et coronariennes. Des études in vitro ont montré que $P$. gingivalis avait la capacité via ses fimbriae d'envahir les cellules endothéliales [17] et que ce phénomène était nécessaire à la progression de l'athérosclérose [18]. Parallèlement, plusieurs modèles animaux d'athérosclérose montrent que $P$. gingivalis pourrait favoriser le développement de plaques d'athérome en interférant avec le profil lipidique et avec la prolifération des cellules musculaires lisses, et ce dans un contexte hypercholestérolémique ou non $[18,19,20]$. Cela suggère que $P$. gingivalis pourrait contribuer à la progression de l'athérothrombose indépendamment de taux élevés de cholestérol (facteur de risque majeur). Des modèles expérimentaux d'anévrisme de l'aorte abdominale ont aussi montré que $P$. gingivalis augmentait la taille de la lésion et favorisait le recrutement des neutrophiles, ce qui pouvait expliquer la non cicatrisation du thrombus luminal, comme dans la pathologie humaine [21]. De plus, les études animales montrent que les souches mutantes de $P$. gingivalis n'accélèrent pas le développement des lésions athéromateuses par comparaison avec les souches sauvages [18]. Les preuves d'une implication directe des bactéries parodontales dans l'athérogènes et l'évolution vers des complications cliniques sont de plus en plus nombreuses. Cependant, pour répondre entièrement aux postulats 
de Koch modifiés, la preuve du potentiel athérogène de bactéries parodontales cultivées à partir de plaques d'athérome humaines fait toujours défaut [11]. La dysfonction endothéliale, marqueur précoce de progression de l'arthérosclérose, est une des variables de substitution utilisées pour évaluer les bénéfices du traitement parodontal sur l'évolution des MCV. L'endothélium activé par les facteurs de risque comme la dyslipidémie, le tabac, le diabète et les infections est rendu perméable et propice au développement des plaques d'athérome [22]. La dysfonction endothéliale est évaluée de façon non invasive par la mesure ultrasonore de la vasorelaxation de l'artère brachiale ou FMD pour flow-mediated dilation. Différentes études ont évalué l'impact du traitement parodontal sur la dysfonction endothéliale [23]. Tonetti et al. [24] Ont montré, dans une étude clinique randomisée portant sur 121 patients, une amélioration de $2 \%$ de la fonction endothéliale à 6 mois avec un traitement parodontal actif (détartrage-surfaçage, avulsions et minocycline en local) en comparaison au détartrage supragingival seul [24]. D'autres facteurs de risque (dyslipidémie, hypertension) et variables de substitution cliniques et biologiques comme l'épaisseur intima-média (IMT pour intima-média thickness) des carotides, les facteurs prothrombotiques, le stress oxydant, les HSP, les cytokines, les MMP ou la pression artérielle ont été étudiés. Le traitement des maladies parodontales semble améliorer faiblement la pression artérielle, le taux de leucocytes, de fibrinogènes, de TNF- $\alpha$, d'E-sélectine, de facteur de Von Willebrand, de MMP, de stress oxydant et semble réduire le taux de CRP. La CRP, protéine hépatique de la phase aigüe de l'inflammation, est associée à un risque faible de MCV si son taux est de $1 \mathrm{mg} / \mathrm{ml}$, alors qu'elle est associée à un risque de MCV multiplié par deux si son taux est au-dessus de $3 \mathrm{mg} / \mathrm{ml}$. Différentes méta-analyses ont confirmé une baisse de la CRP après traitement parodontal de 0,2 à 0,5 $\mathrm{mg} / \mathrm{ml}[25,26]$. Enfin, une étude réalisée sur plus de 11000 sujets âgés de 50 ans a montré qu'une mauvaise hygiène orale était associée à un risque augmenté de $70 \%$ de survenue d'une maladie cardiovasculaire [27]. Il semble que le traitement parodontal, bien qu'entrainant à court terme une réaction inflammatoire, réduise les taux systématiques de marqueurs inflammatoires et améliore la dysfonction endothéliale, à moyen terme. Une récente méta-analyse démontre que le traitement parodontal améliore la fonction endothéliale et diminue le taux des biomarqueurs de la maladie athéromateuse, en particulier chez les sujets souffrant déjà de MCV et /ou de diabète [28]. IGARI K et al. En 2016 ont publié une étude dont le but était d'observer les effets directs des bactéries buccales, telles que Porphyromonas gingivalis $(\mathrm{Pg})$ et Treponema denticola (Td), sur le système vasculaire périphérique. Selon eux ces effets existent mais d'autres études sont nécessaires pour étudier ces corrélations. COTTI E et al. En 2015 ont publié une étude pour évaluer le lien potentiel entre la fonction de la parodontite apicale (AP) et la fonction cardiovasculaire $(\mathrm{CV})$, ils ont mesuré les marqueurs d'inflammation, la réserve d'écoulement endothélial (EFR) et les niveaux de diméthylarginine asymétrique (ADMA), l'inhibiteur endogène de l'oxyde nitrique synthase (NOS) Chez les jeunes adultes âgés de 20 à 40 ans des deux sexes. Ils ont conclu que la présence d'une inflammation chronique chez les jeunes adultes atteints d'AP peut provoquer un dysfonctionnement endothélial précoce documenté par l'EFR réduit. L'AP chez les hommes peut influer sur le métabolisme de la NOS, alors que chez les femmes, il semble impliquer un mécanisme nuisible plus direct. Cette différence dépend de la relation sexuelle et peut être attribuable à l'action protectrice des œstrogènes chez les femmes. JOHANSSON et al. en 2014 ont publié une étude qui examine si l'évaluation préalable du statut parodontal chez les patients atteints d'une maladie coronarienne établie (DAC) peut prédire les futurs points d'évaluation CAO (infarctus du myocarde, nouvelle procédure de revascularisation ou décès lié à la DAC) avec un suivi de 8 ans et si les changements dans le statut parodontal au fil du temps diffèrent chez les patients atteints de DAO comparativement à des témoins sains. Les résultats de cette étude n'ont pas montré d'association significative pendant 8 ans parmi les points d'évaluation CA et l'état parodontal à la ligne de base. D'autres études prospectives à long terme sont nécessaires pour montrer si la parodontite peut être considérée comme un facteur de risque ou de pronostic pour la $\mathrm{CAO}$, en termes d'extrémités, y compris l'infarctus du myocarde, la nouvelle procédure de revascularisation et de décès lié à la DAC. PASQUALINI et al. en 2012 ont publié une étude qui a évalué les associations entre l'état de santé buccal clinique, les polymorphismes CD14 et la CHD. Les sujets souffrant de maladie coronarienne ont une prévalence plus élevée des maladies bucco-dentaires et une plus faible conformité aux stratégies de prévention buccodentaire que les témoins sains. Ils ont conclu que les maladies bucco-dentaires chroniques peuvent augmenter le risque de maladie coronarienne et peuvent constituer un facteur de risque non conventionnel pour la maladie coronarienne. NAKANO et al. en $\mathbf{2 0 0 9}$ ont analysé la répartition des espèces bactériennes par voie orale dans les spécimens cardiovasculaires les bactéries buccales, $\mathrm{y}$ compris les agents pathogènes cariogènes et parodontaux, sont des facteurs étiologiques dans le développement de maladies cardiovasculaires. Ils 
ont conclu que leurs résultats suggèrent que des espèces bactériennes orales spécifiques, telles que $S$. mutans et A. Actinomycetecomitans, sont liées à la bactériémie et peuvent être des facteurs étiologiques pour le développement de maladies cardiovasculaires. SPAHR et al. en $\mathbf{2 0 0 6}$ ont étudié l'association possible entre la parodontite et la maladie coronarienne (CHD), axée sur les aspects microbiologiques. Au total, 789 sujets (263 patients atteints de CHD stable confirmée par angiographie et 526 témoins basés sur la population, contrôlés par l'âge et le sexe sans antécédents de CHD) ont été inclus dans l'étude Coronary Event and Periodontal Disease (CORODONT). Des échantillons de biofilms sous-gingivales ont été analysés pour les agents pathogènes parodontaux Actinobacillus actinomycetemcomitans, Tannerella forsythensis, Porphyromonas gingivalis, Prevotella intermedia et Treponema denticola en utilisant l'hybridation ADN-ADN. La nécessité d'un traitement parodontal chez chaque sujet a été évaluée à l'aide de l'indice communautaire des besoins de traitement (CPITN). Les principales mesures de résultats incluaient le fardeau pathologique partagonal total, le nombre de divers agents pathogènes parodontaux dans le biofilm sous-gingival et les besoins de traitement parodontal (selon le CPITN). Ils ont conclu que leurs résultats suggèrent une association entre la parodontite et la présence de CHD. Le fardeau pathologique parodontal, et en particulier l'infection par A Actinomycetecomitans, peut-être d'une importance particulière.

\section{CONCLUSION}

Avec une étiologie infectieuse pour les maladies parodontales et diverse pour les maladies cardiovasculaires, un lien a été établi entre les deux maladies dans diverses études épidémiologiques et cliniques mais toutefois reste controverse dans d'autres études. Les bactériémies associées aux parodontites sont responsables d'une activation de la réponse immuno-inflammatoire favorisant la formation, la maturation et la complication des plaques d'athérome. Il existe un risque accru de MCV chez les patients atteints de parodontite chronique, indépendamment des autres facteurs de risques cardiovasculaires. Le traitement parodontal réduit le niveau d'inflammation systémique et améliore la fonction endothéliale, mais ne modifie pas la dyslipidémie.

\section{Conflit d'intérêt : Aucun}

\section{REFERENCES}

1-BERCY P. TENENBAUM H. Parodontologie: du Diagnostique à la Pratique. Paris : DE BOECK SUPERIEUR; 1996, 296p.

2- BAHEKAR AA, SINGH S, SAHA S.The prevalence and incidence of coronary heart disease is significantly increased in periodontitis: a metaanalysis. Am Heart J, 2007, 154 : 830-837.

3-HUMPHREY LL, FU R, BUCKLEY DI. Periodontal disease and coronary heart disease incidence: a systematic review and meta-analysis. J. Gen Intern Med, 2008, 23: 2079-2086

4-KHADER YS, ALBASHAIREH ZS, ALOMARI MA. Periodontal diseases and the risk of coronary heart and cerebrovascular diseases: a meta-analysis. J. Periodontol, 2004, 75: 1046-1053.

5-MUSTAPHA IZ, DEBREY S, OLADUBU M, UGARTE R. Markers of systemic bacterial exposure in periodontal disease cardiovascular disease risk: a review and meta-analysis. J. Periodontol, 2007, 78: 2289-2302.

6-MATTILA KJ, NIEMINEN MS, VALTONEN VV. Association between dental health and acute myocardial infarction. BMJ, 1989, 298: 779-981

7-LOCKHART PB, BOLGER AF, PAPAPANOU PN. Periodontal diseases and atherosclerotic vascular disease: does the evidence support an indépendent association? : scientific statement from the American heart association. Circulation, 2012, $125: 2520-2544$.

8-DIETRICH T, SHARMA P, WALTER C. The epidemiological evidence behind the association between periodontitis and incident atherosclerotic cardiovascular diseases. J Clin Periodontol, 2013, 40: S70-S84.

9-SCHENKEIN HA, LOOS BG.Inflammatory mechanisms linking periodontal diseases to cardiovascular diseases. J. Clin Periodontol, 2013, 40: S51-S69.

10-MICHEL JB, VIRMANI R, ARBUSTINI E, PASTERKAMP G. Intraplaque haemorrhages as the trigger of plaque vulnerability.

Eur Heart J, 2011, 32: 1977-1985.

11-REYES L, HERRERA D, KOZAROV E.Periodontal bacterial invasion and infection: contribution to atherosclerotic pathology. J Clin Periodontol, 2013, 40: S30-S50.

12-TOMAS I, DIZ P, TOBIAS A.Periodontal heath status and bacteraemia from daily oral activities: systematic review / meta-analysis. J. Clin Periodontol, 2012, 39: 213-228.

13-HARASZTHY VI, ZAMBON JJ, TREVISAN M, ZEID M.Identification of periodontal pathogens in atheromatous plaques. J, Periodontol 2000, 71: 1554-1560.

14-OHKI T, ITABASHI Y, KOHNO T.Detection of periodontal bacteria in thrombi of patients with acute myocardial infarction by polymerase chain reaction. Am Heart J. 2012, 163(2): 164-167.

15-KOZAROV EV, DORN BR, SHELBOURNE CE.Human atherosclerotic plaque contains viable invasive actinobacillus actinomycetemcomitens and Porphyromonas gingivalis. Arterioscler Thromb Vasc Biol, 2005, 25: el 7-8. 
16-RAFFERTY B, JONSSON D, KALACHIKOV S.Impact of monocytic cells on recovery of uncultivable bacteria from atherosclerotic lesions. J Intern Med, 2011, 270: 273-280.

17-DESHPANDE RG, KHAN MB, GENCO CA.

Invasion of aortic and heart endothelial cells by Porphyromonas gingivalis, infec Immun, 1998, 66: 5337-5343.

18-AMAR S, WU SC, MADAN M. Is Porphyromonas gingivalis cell invasion required for atherogenesis? pharmacotherapeutic implications. J. Immunol, 2009, 182 : 1584-1592.

19-LALLA E, LAMSTER IB, HOFMANN MA.Oral infection with a periodontal pathogen accelerates early atherosclerosis in apolipoprotein E-null mice. Arterioscler thromb Vasc Biol, 2003, 23: 1405-1411.

20-LI L, MESSAS E, BATISTA EL Jr. Porphyromonas gingivalis infection accelerates the progression of atherosclerosis in a heterozygous apolipoprotein E-deficient murine model. Circulation, 2002, 105: 861-867.

21-DELBOSC S, ALSAC JM, JOURNE C.Porphyromonas gingivalis participates in pathogenesis of human abdominal aortic aneurysm by neutrophil activation. Proof of concept in rats. PLos One, 2011, 6: el 8679.

22-LIBBY P. Inflammation in atherosclerosis. Nature, 2002, 420: 868-874.

23-D'AIUTO F, ORLANDI M, GUNSOLLEY JC. Evidence that periodontal treatment improves biomarkers and CVD outcomes. J Clin Periodontol, 2013, 40: S85-S105.

24-TONETTI MS, D'AIUTO F, NIBALI L.Treatment of periodontitis and endothelial function. N Engl J Med, 2007, 356: 911-920.

25-FREITAS C, GOMES-FILHO IS, NAVES RC.Influence of periodontal therapy on C-reactive protein level: a systematic review and metaanalysis. J Applied Oral Scien, 2012, 20: 1-8.

26-IOANNIDOU E. MALEKZADETH T, DONGARI-BAGTZOGLOU A. Effect of periodontal treatment on serum C-réactive protein levels: a systemetic review and meta-analysis. J. Periodontol 2006, 77: 1635-1642.

27-OLIVIERA DE C, WATT R, HAMER M.Toothbrushing, inflammation, and risk of cardiovascular diseases: results from scotrish Hearlth survey. BMJ, 2010, 27, 340: C2451.

28-TEEUW WJ, SLOT DE, SUSANTO H.Treatment of periodontitis improves the atherosclerotic profile: a systematic review and meta-analysis. J. Periodontol, 2014, 41: 70-79.

29-IGARI K, INOUE Y, IWAI T.An Experimental Model of Peripheral Vascular Disease Involving the Intravenous Injection of Oral Bacteria. Ann Vasc Dis. 2016;9(4):267-271.
30-COTTI E, ZEDDA A, DEIDDA M, PIRAS $A$, FLORE G, IDEO $F$ et al.Endodontic infection and endothelial dysfunction are associated with different mechanisms in men and women. $\mathbf{J}$ Endod. 2015;41(5):594-600.

31-JOHANSSON CS, RAVALD N, PAGONIS C, RICHTER A. Periodontitis in patients with coronary artery disease: an 8-year follow-up.

J Periodontol. 2014;85(3):417-25.

32-PASQUALINI D, BERGANDI L, PALUMBO L, BORRACCINO A. Association among oral health, apical periodontitis, CD14 polymorphisms, and coronary heart disease in middle-aged adults. J Endod. 2012 Dec;38(12):1570-7.

33-COTTI E, DESSÌ C, PIRAS A, FLORE G, DEIDDA M, MADEDDU C. Association of endodontic infection with detection of an initial lesion to the cardiovascular system. J Endod. 2011;37(12):1624-9.

34-NAKANO K, NEMOTO H, NOMURA $R$, INABA H. Detection of oral bacteria in cardiovascular specimens. Oral Microbiol Immunol. 2009; 24(1):64-8.

35-SPAHR A, KLEIN E, KHUSEYINOVA $\mathrm{N}, \mathrm{BOECKH} \quad \mathrm{C}$, MUCHE R, KUNZE M. Periodontal infections and coronary heart disease: role of periodontal bacteria and importance of total pathogen burden in the Coronary Event and Periodontal Disease (CORODONT) study. Arch Intern Med. 2006;166 (5):554-9. 